\title{
Induction of the Type I IFN Response by Human Metapneumovirus Lacking SH, G, or M2.2 Expression ${ }^{+}$
}

\author{
Kevin Groen *, Stefan van Nieuwkoop, Ron Fouchier and Bernadette van den Hoogen \\ Department of Viroscience, Erasmus MC, Doctor Molenwaterplein 40, 3015 GD Rotterdam, The Netherlands; \\ s.vannieuwkoop@erasmusmc.nl (S.v.N.); r.fouchier@erasmusmc.nl (R.F.); \\ b.vandenhoogen@erasmusmc.nl (B.v.d.H.) \\ * Correspondence: k.groen@erasmusmc.nl; Tel.: +31-0107043330 \\ † Presented at Viruses 2020-Novel Concepts in Virology, Barcelona, Spain, 5-7 February 2020.
}

Published: 11 August 2020

\begin{abstract}
The human metapneumovirus (HMPV), a member of the Pneumoviridae family, is a major cause of respiratory illness, primarily in young children, the elderly, and immunocompromised individuals. Having a fundamental understanding of the viral evasion of innate immune responses is crucial for the rational design of antiviral therapies. Several studies have reported on how HMPV subverts innate immune responses, with roles for $\mathrm{SH}, \mathrm{G}$, and M2.2 proteins. However, these studies often conflict. It has also been reported that eliminating the M2.2 ORF results in insertions and deletions around the M2.2 ORF, which could result in an M2.2-independent interaction with the immune system. We aimed to investigate how HMPV interacts with the innate immune response. Therefore, recombinant viruses lacking M2.2, SH, or G protein expression were generated either by deletion or by ablation of protein expression through mutations. Phenotypic analysis revealed that viruses lacking M2.2 expression are attenuated on interferon-competent A549 cells, but not on interferon-deficient cells. Deletion of ORFs compared to ablation of expression through mutations did not result in differences in replication kinetics. Viruses lacking M2.2 expression induced interferon- $ß$ protein production, indicating interferon-antagonistic functions of the M2.2 protein, as previously reported. Phenotypic analysis of A549 cells knocked out for RIG-I, MAVS, and PKR revealed the role of RIG-I in the immune response towards HMPV. Next-generation sequencing analysis of viruses lacking M2.2 expression but not G or SH expression showed hypermutation throughout the virus genome. The hypermutation patterns suggest a role for adenosine deaminase acting on RNA (ADAR) editing. We addressed the question of whether RIG-I activation by viruses lacking M2.2 expression is due to hypermutated genomes or the absence of M2.2 as an interferon antagonist. Additionally, we investigated the role of ADAR in HMPV infection. We present our data on the possible influence of ADAR in HMPV infection by next-generation sequencing of viral stocks in cell knockdowns of ADAR generated by CRISPR-interference.
\end{abstract}

Keywords: human metapneumovirus; type-I IFN; ADAR

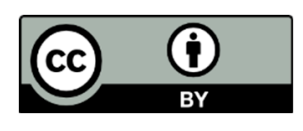

(C) 2020 by the authors. Licensee MDPI, Basel, Switzerland. This article is an open access article distributed under the terms and conditions of the Creative Commons Attribution (CC BY) license (http://creativecommons.org/licenses/by/4.0/). 